\title{
A space-frequency multiplicative regularization for force reconstruction problems
}

\author{
M. Aucejo ${ }^{\mathrm{a}}$, O. De Smet $^{\mathrm{a}}$ \\ ${ }^{a}$ Structural Mechanics and Coupled Systems Laboratory, Conservatoire National des Arts \\ et Métiers, 2 Rue Conté, 75003 Paris, France
}

\begin{abstract}
Dynamic forces reconstruction from vibration data is an ill-posed inverse problem. A standard approach to stabilize the reconstruction consists in using some prior information on the quantities to identify. This is generally done by including in the formulation of the inverse problem a regularization term as an additive or a multiplicative constraint. In the present article, a space-frequency multiplicative regularization is developed to identify mechanical forces acting on a structure. The proposed regularization strategy takes advantage of one's prior knowledge of the nature and the location of excitation sources, as well as that of their spectral contents. Furthermore, it has the merit to be free from the preliminary definition of any regularization parameter. The validity of the proposed regularization procedure is assessed numerically and experimentally. It is more particularly pointed out that properly exploiting the space-frequency characteristics of the excitation field to identify can improve the quality of the force reconstruction.
\end{abstract}

Keywords: Inverse problem, Force reconstruction, Multiplicative regularization, Mixed norms.

${ }^{*}$ Corresponding author. E-mail address: mathieu.aucejo@cnam.fr 


\section{Introduction}

The characterization of dynamic forces acting on a structure remains one of the major industrial concerns to control broadband excitation sources or establish consistent excitation models for numerical simulation and design of complex structures. Unfortunately, the direct measurement of excitation forces is generally difficult or even impossible in practical situations. The basic idea to circumvent this practical limitation is to perform an indirect measurement from related accessible quantities such as displacement, velocity or acceleration fields.

Such techniques, referred to as force reconstruction problems, belong to the class of ill-posed inverse problems, meaning that the existence of a unique stable solution is not guaranteed. A possible solution to remedy this undesirable feature consists in including in the reconstruction problem some prior information on the forces to identify to constrain the space of admissible solutions. The mathematical transcription of this simple idea leads to express the inverse problem as a minimization problem, where prior information on the excitation forces is encoded in a regularization term. This regularization term can be incorporated in the formulation as an additive constraint, given

rise to Tikhonov-like regularizations [1]. It should, however, be noted that a proper choice of the regularization term is crucial since it strongly conditions the quality of the reconstruction.

In general, two categories of reconstruction problem can arise in practical situations. The first one is related to the localization of excitation sources, while the second one consists in reconstructing the frequency spectrum or the 
time signal of prelocalized sources. Regarding the localization problem, the regularization term only reflects the spatial prior information on the sources to identify. It is often expressed as the $\ell_{p}$-norm of the desired solution vector. Such a norm is highly flexible to express one's prior knowledge on the nature of the forces to reconstruct [2], since smooth solutions are obtained for $p=2[1,3,4]$, while sparse excitation fields are promoted for $p \leq 1$ $[5,6,7,8,9]$. It should be noticed that the spatial prior information is classically defined in a global manner, meaning that all the sources exciting the structure have the same attribute, i.e. localized, distributed or piecewise continuous. Such global regularization terms lead to poor reconstructions, if the actual excitation field combines sources of different nature, since the a priori has to reflect a compromise between contradictory distributions [2]. To bypass this difficulty, a group regularization term, defined from $\ell_{p}$-norms, has been recently proposed to exploit local spatial prior information on both the nature and the location of excitation sources $[10,11]$. In all the aforementioned methods developed in the frequency domain, the reconstruction problem is solved frequency by frequency, which is equivalent to suppose that the frequency spectrum of the identified sources is discontinuous. This lack of spectral continuity is inherent to these approaches and can induce potential inaccuracies in the reconstructed frequency spectrum if the sources are broadband [12]. On the other hand, for reconstructing the frequency spectrum or the time signal of prelocalized sources, the regularization term has to reflect prior information on the nature of the excitation signal. Since the sources are usually broadband, the force signal exhibits a certain continuity. That is why, the corresponding regularization term is generally constructed from the 
$\ell_{2}-$ norm of the solution vector to identify $[13,14,15,16]$. Obviously, such identification methods are not suited for source localization and should fail when the locations of the potential sources does not match the actual ones. Consequently, it appears that the vast majority of the methods proposed in the literature are generally unable to consistently tackle both localization and spectral/temporal reconstruction problems at the same time. To the best of our knowledge, only a few methods have been developed to address these issues. However, they are often limited to the reconstruction of point sources or to configurations where the spatial distribution of the sources and the nature of the excitation signals share the same characteristics such as the sparsity $[17,18,19,20,21]$ or address the space-time (or space-frequency) reconstruction problem in a separated manner [22].

It is thus of primary interest to simultaneously exploit both the spatial and the spectral/temporal features of excitation sources to aid the reconstruction process in finding the best possible solution. These requirements are actually satisfied by regularization terms derived from a mixed $\ell_{p, q}$-norm. A mixed norm is a matrix norm defined for any matrix $\mathbf{F}$ by the following relation:

$$
\left.\|\mathbf{F}\|_{p, q}=\left[\sum_{i}\left(\sum_{j}\left|F_{i j}\right|^{p}\right)^{\frac{q}{p}}\right]^{\frac{1}{q}} \forall(p, q) \in\right] 0,+\infty\left[^{2} .\right.
$$

Mixed $\ell_{2, q}-$ norms, for $q \leq 1$, have revealed their suitability in signal and image recovery applications $[23,24,25,26]$. In the context of force identification, Rezayat et al. first derive a Tikhonov-like regularization using a regularization term based on a mixed $\ell_{2,1}-$ norm to reconstruct broadband point forces $[12,21]$. 
In the present paper, an original regularization strategy is developed to solve both localization and spectral reconstruction problems within a unique framework. More precisely, the proposed approach first relies on the definition of a regularization term that properly reflects one's prior knowledge on the type (localized or distributed) of the excitation forces, as well as on the nature of the excitation signal. From a mathematical standpoint, this regularization term is constructed from the general mixed $\ell_{p, q}-$ norm. Then, to derive the generic form of the identification problem, the proposed regularization term is included in the formulation as a multiplicative constraint. In doing so, a particular form of multiplicative regularization is obtained. This regularization strategy, originally developed by Van den Berg et al. [27], has several advantages compared to the more classical additive approaches, which explains its use in the present article. It has, in particular, the merit to be free from the preliminary definition of any regularization parameter. Accordingly, it is generally faster than the related Tikhonov-like regularization, but leads to similar reconstructed solutions [28]. To clearly highlight and explain the main features of the proposed regularization strategy, this article is divided into five parts. In section 2, the reconstruction model used for deriving the space-frequency regularization is detailed. Section 3 is devoted to the description of the generic formulation of the regularization problem. Its resolution is performed from an iterative procedure introduced in section 4 . Numerical and experimental validations of the space-frequency regularization are respectively proposed in sections 5 and 6 . Obtained results point out the practical and potential interest in exploiting both spatial and spectral prior information for improving the quality of the force reconstruction. Finally, 
the last part of this paper introduces the theoretical basis of the possible extension of the space-frequency regularization to time domain applications.

\section{Description of the reconstruction model}

The definition of the space-frequency reconstruction problem requires the construction of a model describing the dynamic behavior of the structure and relating the measured vibration field to the excitation field to identify. To this end, let us consider the general situation where the studied structure is supposed linear and time-invariant. In this case, the dynamic behavior of the structure at a particular frequency $f_{j}$ is completely described by the transfer functions matrix $\mathbf{H}\left(f_{j}\right)$. Depending on the method used to derive this transfer function matrix, two reconstruction models can be defined. Indeed, if $\mathbf{H}\left(f_{j}\right)$ is measured, the reconstruction model is written [29, 30, 31]:

$$
\mathbf{X}\left(f_{j}\right)=\mathbf{H}\left(f_{j}\right) \mathbf{F}\left(f_{j}\right), \quad \forall j=1, \ldots, N
$$

where $\mathbf{X}\left(f_{j}\right)$ is the measured vibration field and $\mathbf{F}\left(f_{j}\right)$ is the unknown excitation field at frequency $f_{j}$ and $N$ is the number of studied frequencies.

On the other hand, if $\mathbf{H}\left(f_{j}\right)$ is numerically computed, the reconstruction model is then defined by the following relation [32, 33]:

$$
\mathbf{X}\left(f_{j}\right)=\mathbf{H}\left(f_{j}\right) \mathbf{F}\left(f_{j}\right)+\mathbf{N}\left(f_{j}\right) \quad \forall j=1, \ldots, N
$$

where $\mathbf{N}\left(f_{j}\right)$ is the noise vector reflecting measurement and modeling errors.

To implement the proposed space-frequency regularization, one has to define a global model from the reconstruction model given by Eq. (2) or (3) 
at each studied frequency. Considering that the transfer function matrices are obtained from FEM, the global reconstruction model is written [21]:

$$
\overline{\mathbf{X}}=\overline{\mathbf{H}} \overline{\mathbf{F}}+\overline{\mathbf{N}}
$$

where $\overline{\mathbf{H}}=\operatorname{diag}\left[\mathbf{H}\left(f_{1}\right), \ldots, \mathbf{H}\left(f_{j}\right), \ldots, \mathbf{H}\left(f_{N}\right)\right]$ is the global transfer functions matrix, $\overline{\mathbf{X}}=\left[\mathbf{X}\left(f_{1}\right)^{T}, \ldots, \mathbf{X}\left(f_{j}\right)^{T}, \ldots, \mathbf{X}\left(f_{N}\right)^{T}\right]^{T}$ is the global measured vibration field, $\overline{\mathbf{F}}$ is the global unknown excitation field and $\overline{\mathbf{N}}$ is the global noise vector.

\section{Space-frequency multiplicative regularization}

As already evoked in the introduction, a classical approach to stabilize an inverse problem consists in including in the formulation of the problem some prior information on the forces to identify. For this purpose, the inverse problem is generally expressed as a constrained minimization problem, namely:

$$
\min _{\overline{\mathbf{F}}} \mathcal{F}(\overline{\mathbf{X}}-\overline{\mathbf{H}} \overline{\mathbf{F}}) \quad \text { subject to } \quad \mathcal{R}(\overline{\mathbf{F}}) \leq \beta,
$$

where:

- $\mathcal{F}(\overline{\mathbf{X}}-\overline{\mathbf{H}} \overline{\mathbf{F}})$ is the data-fidelity term which controls the a priori on the noise corrupting the data $[34,35,36]$;

$-\mathcal{R}(\overline{\mathbf{F}})$ is the regularization term that encodes prior information on the force distribution $\overline{\mathbf{F}}[5,37,38]$;

- $\beta$ is some positive constant related to the solution variance. 
For simplifying the resolution of the reconstruction problem, the constrained minimization problem is generally reformulated into an unconstrained form, in which the regularization term appears as an additive or a multiplicative constraint. The additive formulation actually corresponds to a Tikhonov-like regularization, that is:

$$
\overline{\mathbf{F}}_{\mathbf{a}}=\underset{\overline{\mathbf{F}}}{\operatorname{argmin}} \mathcal{F}(\overline{\mathbf{X}}-\overline{\mathbf{H}} \overline{\mathbf{F}})+\lambda \mathcal{R}(\overline{\mathbf{F}})
$$

where $\lambda$ is a Lagrange multiplier, a.k.a. the regularization parameter, which controls the trade-off between the data-fidelity and regularization terms.

The main issue of such a formulation is related to the choice of the regularization parameter. Generally, the optimal value of this parameter is automatically selected from adapted numerical procedures [39, 40]. However, these selection methods are often computationally intensive and time-consuming. To circumvent this drawback, the multiplicative strategy can be used as an alternative [41, 42, 43, 44]. Formally, this approach simply writes:

$$
\overline{\mathbf{F}}_{\mathbf{m}}=\underset{\overline{\mathbf{F}}}{\operatorname{argmin}} \mathcal{F}(\overline{\mathbf{X}}-\overline{\mathbf{H}} \overline{\mathbf{F}}) \cdot \mathcal{R}(\overline{\mathbf{F}}) .
$$

From a practical standpoint, this regularization method eliminates the need for the selection of the regularization parameter. As a result, the multiplicative strategy is significantly faster than the additive formulation. It should however be noted that both techniques lead to similar identified excitation fields [11]. For this particular reason, the proposed space-frequency regularization method is based on a multiplicative approach. The choice of the data-fidelity and regularization terms is detailed in the following subsections. 


\subsection{Definition of the regularization term}

An appropriate definition of the regularization term is all the more crucial since it conditions the quality of the reconstructed solution $[2,3,11,20]$. In this paper, the regularization term is defined from the general mixed $\ell_{p, q}-$ norm in order to properly exploit one's prior knowledge on the spatial and spectral characteristics of the forces to reconstruct. To highlight the main properties of the proposed regularization term and make its definition clearer, a step-by-step description is introduced. More precisely, it consists in explaining the construction of the space-frequency regularization term from the separate definition of frequency and spatial regularization terms.

\subsubsection{Frequency regularization term}

The frequency regularization term has to reflect prior information on the nature of the excitation signal. To this end, let us consider a reconstruction point i located on the structure. For this particular point, the frequency spectrum (a.k.a frequency group) is extracted from the global force vector $\overline{\mathbf{F}}$ and is simply written:

$$
\overline{\mathbf{F}}[i,:]=\left[F_{i}\left(f_{1}\right), \ldots, F_{i}\left(f_{j}\right), \ldots, F_{i}\left(f_{N}\right)\right]^{T}
$$

To take into account a wide class of excitation signals, the frequency regularization term can be expressed from a $\ell_{p}-$ norm, that is:

$$
\mathcal{R}(\overline{\mathbf{F}}[i,:])=\|\overline{\mathbf{F}}[i,:]\|_{p}
$$

Such a regularization term is well adapted to deal with broadband as well as sparse excitation signals. Indeed, when rotating machinery applications or milling processes are considered, the force signal is generally sparse, since 
only a few specific frequencies are excited $[45,46]$. Such a sparsity pattern can be enforced using $p \leq 1$ [7]. On the contrary, when the structure is excited by an impact or a shaker fed by a white noise signal, the force spectrum is necessarily broadband and continuous, i.e. rather smooth. In this situation, it is recommended to set $p=2$ to promote the $C^{0}$ continuity of the force signal. To enforce a particular continuity pattern, such as $C^{n}$ or piecewise continuity, a differentiation operator $\mathbf{L}_{\mathbf{f}}$ has to be introduced to include in the formulation one's prior knowledge on the continuity of force spectrum $[47,48]$. As a result, a general form of frequency regularization term is:

$$
\mathcal{R}(\overline{\mathbf{F}}[i,:])=\left\|\mathbf{L}_{\mathbf{f}}(\overline{\mathbf{F}}[i,:])\right\|_{p} .
$$

Practically, the differentiation operator corresponds to the discretized form of a certain continuum derivative operator. For instance, to promote the $C^{1}$ continuity of the force spectrum, one has to set $p=2$ and define a frequency differentiation operator $\mathbf{L}_{\mathbf{f}}$ of the form:

$$
\mathbf{L}_{\mathbf{f}}=\frac{1}{\Delta f}\left(\begin{array}{cccccc}
-1.5 & 2 & -0.5 & & & \\
-0.5 & 0 & 0.5 & & & \\
& & \ddots & & & \\
& & & \ddots & & \\
& & & -0.5 & 0 & 0.5 \\
& & & 0.5 & -2 & 1.5
\end{array}\right)
$$

where $\Delta f$ is the frequency resolution.

\subsubsection{Spatial regularization term}

The spatial regularization term has to reflect prior information on the type of the excitation forces acting on a structure. To this end, let us consider the 
force vector at a particular frequency $f_{j}$, namely:

$$
\overline{\mathbf{F}}[:, j]=\mathbf{F}\left(f_{j}\right)=\left[F_{1}\left(f_{j}\right), \ldots, F_{i}\left(f_{j}\right), \ldots, F_{M}\left(f_{j}\right)\right]^{T},
$$

where $M$ is the number of reconstruction points.

To derive a consistent spatial regularization term, it has to be flexible enough to properly reflect different priors. In this paper, we consider that only one type of sources excites the structure. Yet, the derivations presented in the next sections can be easily extended to the case where several types of sources act simultaneously on the structure. To this end, the concept of local regularization term has to be introduced [11]. Here, the spatial regularization term is chosen such that:

$$
\mathcal{R}(\overline{\mathbf{F}}[:, j])=\left\|\mathbf{L}_{\mathbf{s}}(\overline{\mathbf{F}}[:, j])\right\|_{q}^{q},
$$

where $\mathbf{L}_{\mathbf{s}}$ is the spatial differentiation operator that allows controlling the regularity of the solution.

As shown in Refs. $[2,11]$, the proposed regularization term is particularly well adapted for force reconstruction problems, since smooth solutions are promoted for $q=2$ and $\mathbf{L}_{\mathbf{s}}=\mathbf{I}_{M}\left(\mathbf{I}_{M}\right.$ : Identity matrix of dimension $\left.M\right)$, while sparse solutions are favored for $q \leq 1$ and $\mathbf{L}_{\mathbf{s}}=\mathbf{I}_{M}$. For promoting piecewise continuous solutions, one can set $q \leq 1$ and define $\mathbf{L}_{\mathbf{s}}$ as the discretized form of the $n^{\text {th }}$-order differential operator. Thus, the solution is approximated by piecewise constant segments if $n=1$ [see Eq. (11)], while the solution is approximated by piecewise polynomials of degree 1 if $n=2$ [47]. 


\subsubsection{Space-frequency regularization term}

The role of the space-frequency regularization term is to reflect the experimenter's knowledge on both the spatial and spectral characteristics of the forces to identify. From the definition of the frequency and spatial regularization terms, it readily comes:

$$
\mathcal{R}(\overline{\mathbf{F}})=\|\overline{\mathbf{L}} \overline{\mathbf{F}}\|_{p, q}^{q}=\sum_{i=1}^{M}\|(\overline{\mathbf{L}} \overline{\mathbf{F}})[i,:]\|_{p}^{q},
$$

where $\overline{\mathbf{L}}$ is the global space-frequency differentiation operator and the vector $(\overline{\mathbf{L}} \overline{\mathbf{F}})[i]$ is defined by analogy with Eq. (8). For implementation purposes, the space-frequency differentiation operator is expressed:

$$
\overline{\mathbf{L}}=\overline{\mathbf{L}_{\mathbf{s}}} \overline{\mathbf{L}_{\mathbf{f}}}
$$

where $\overline{\mathbf{L}_{\mathbf{s}}}=\mathbf{I}_{N} \otimes \mathbf{L}_{\mathbf{s}}$ and $\overline{\mathbf{L}_{\mathbf{f}}}$ are respectively the global spatial and frequency differentiation operator.

Such a regularization term introduces explicitly a coupling between the coefficients of the vector $\overline{\mathbf{L}} \overline{\mathbf{F}}$ and allows promoting some structures observed in real signals [24]. To illustrate this particular property of mixed norms, let us consider the simple case where $\overline{\mathbf{L}}=\mathbf{I}_{N M}$. Furthermore, if one represents

the force vector $\overline{\mathbf{F}}$ as a matrix, where the rows correspond to the force spectrum at a particular location and the columns to the force field at a specific 
frequency, it comes:

$$
\overline{\mathbf{F}}=\left[\begin{array}{ccccc}
F_{1}\left(f_{1}\right) & \cdots & F_{1}\left(f_{j}\right) & \cdots & F_{1}\left(f_{N}\right) \\
\vdots & & \vdots & & \vdots \\
F_{i}\left(f_{1}\right) & \cdots & F_{i}\left(f_{j}\right) & \cdots & F_{i}\left(f_{N}\right) \\
\vdots & & \vdots & & \vdots \\
F_{M}\left(f_{1}\right) & \cdots & F_{M}\left(f_{j}\right) & \cdots & F_{M}\left(f_{N}\right)
\end{array}\right] .
$$

If we further assume that $(p, q)=(2,1)$, then the matrix $\overline{\mathbf{F}}$ is supposed to be sparse along the lines (space) and full along the rows (frequency). In other words, one promotes the spatial sparsity of the excitation field (localized sources) and the continuity of its frequency spectrum (broadband source signal). Consequently, the present space-frequency regularization term is highly flexible, since it allows dealing with various force distributions and various excitation signals within a unique framework.

\subsection{Definition of the data-fidelity term}

The data-fidelity term is a measure of the difference between the measured vibration field $\overline{\mathbf{X}}$ and the reconstructed vibration field $\overline{\mathbf{H}} \overline{\mathbf{F}}$. Consequently, it reflects prior information on the bias between measured and reconstructed vibration fields. In other words, it defines one's prior knowledge of the noise corrupting the data. A common assumption consists in considering that the vibration field is corrupted at each frequency by an additive Gaussian white noise. In this situation, the data-fidelity term is usually expressed as:

$$
\mathcal{F}(\overline{\mathbf{X}}-\overline{\mathbf{H}} \overline{\mathbf{F}})=\|\overline{\mathbf{X}}-\overline{\mathbf{H}} \overline{\mathbf{F}}\|_{2}^{2}
$$




\subsection{Generic form of the space-frequency multiplicative regularization}

The generic form of the proposed space-frequency multiplicative regularization is simply obtained by introducing Eqs. (14) and (17) into Eq. (7). In doing so, it readily comes:

$$
\overline{\mathbf{F}}_{\mathbf{m}}=\underset{\overline{\mathbf{F}}}{\operatorname{argmin}}\|\overline{\mathbf{X}}-\overline{\mathbf{H}} \overline{\mathbf{F}}\|_{2}^{2} \cdot\|\overline{\mathbf{L}} \overline{\mathbf{F}}\|_{p, q}^{q} .
$$

\section{Resolution of the regularization problem}

As already stated in section 3, the proposed formulation is flexible enough to properly incorporate prior information on the type of the sources acting on the structure, but also on the nature of the excitation signals. In return, the functional to minimize is generally non-convex, implying that the corresponding solution, if exists, has no closed-form expression. For this primary reason, the implementation of an iterative procedure is the keystone to obtain consistent regularized solutions. The requested algorithm should be easy to implement, sufficiently general to cope with a wide range of configurations depending on the values of $p$ and $q$ and should exhibit a linear or super-linear rate of convergence. All these requirements are met by the Iteratively Reweighted Least Squares algorithm [49, 50, 51, 52]. Such an iterative procedure has been successfully applied for solving force reconstruction problems at a particular frequency [2, 11, 28]. In the next of this section, an adapted IRLS algorithm is derived to deal with the proposed space-frequency multiplicative regularization. 


\subsection{Basic principle}

The proposed IRLS algorithm is a fixed-point algorithm adapted for solving minimization problems of the form of Eq. (18). The underlying idea behind this algorithm is to define a fixed-point iteration having a unique and explicit solution. In doing so, it is expected to recover the solution of the original minimization problem when the convergence of the iterative process is reached. To derive the desired fixed-point iterate, the mixed $\ell_{p, q}-$ norm to the power of $q$ is recast into a squared weighted $\ell_{2}$-norm. In doing so, one obtains at iteration $\mathrm{k}+1$ :

$$
\overline{\mathbf{F}}_{\mathbf{m}}^{(k+1)}=\underset{\overline{\mathbf{F}}}{\operatorname{argmin}}\|\overline{\mathbf{X}}-\overline{\mathbf{H}} \overline{\mathbf{F}}\|_{2}^{2} \cdot\left\|\overline{\mathbf{W}}^{(k)^{1 / 2}} \overline{\mathbf{L}} \overline{\mathbf{F}}\right\|_{2}^{2},
$$

where $\overline{\mathbf{F}}_{\mathbf{m}}^{(k+1)}$ is the solution at iteration $\mathrm{k}+1$ and $\overline{\mathbf{W}}^{(k)}$ is a global weighting diagonal matrix defined as a function of $\overline{\mathbf{L}} \overline{\mathbf{F}}_{\mathbf{m}}^{(k)}$. In the present case, the coefficients of the global weighting matrix are defined such that:

$$
\overline{\mathrm{W}}_{I}^{(k)}=W_{i}^{s(k)} \cdot W_{i, j}^{f(k)},
$$

where $I=j+N(i-1)$ is a global index (for $i=1, \ldots, M$ and $j=1, \ldots, N)$ and $W_{i}^{s(k)}$ and $W_{i, j}^{f(k)}$ are the weighting coefficients related to the space $(s)$ and the frequency $(f)$ domains.

By setting $\overline{\mathbf{Y}}[i,:]=\left(\overline{\mathbf{L}} \overline{\mathbf{F}}_{\mathbf{m}}^{(k)}\right)[i,:]$, the weighting coefficients $W_{i, j}^{f(k)}$ and $W_{i}^{s(k)}$ are written [see Appendix A]:

$$
W_{i, j}^{f(k)}=\max \left(\epsilon^{p-2},\left|Y_{i}\left(f_{j}\right)\right|^{p-2}\right)
$$

and

$$
W_{i}^{s(k)}=\max \left(\left[\epsilon^{2}\right]^{\frac{q}{p}-1},\left[\left\|\mathbf{W}_{\mathbf{i}}^{\mathbf{f}(k)^{1 / 2}} \overline{\mathbf{Y}}[i,:]\right\|_{2}^{2}\right]^{\frac{q}{p}-1}\right)
$$


where $\mathbf{W}_{\mathbf{i}}^{\mathbf{f}(k)}=\operatorname{diag}\left[W_{i, 1}^{f(k)}, \ldots, W_{i, N}^{f(k)}\right]$ and $\epsilon$ is a damping parameter that allows avoiding infinite weights. The damping parameter is automatically selected once for all at the beginning of the iterative process from the cumulative histogram of $\left|\overline{\mathbf{L}}_{\mathbf{\mathbf { m }}}^{(0)}\right|[2,53]$. Actually, its value is calculated so that $5 \%$ of the values $\left|\overline{\mathbf{L}} \overline{\mathbf{F}}_{\mathbf{m}}^{(0)}\right|$ are less than or equal to $\epsilon$.

To have a better insight on the regularization properties of the multiplicative strategy, the operational form of the previous minimization problem should be given. After some simple calculations, one finds the following explicit relation:

$$
\overline{\mathbf{F}}_{\mathbf{m}}^{(k+1)}=\left(\overline{\mathbf{H}}^{H} \overline{\mathbf{H}}+\alpha^{(k+1)} \overline{\mathbf{L}}^{H} \overline{\mathbf{W}}^{(k)} \overline{\mathbf{L}}^{-1} \overline{\mathbf{H}}^{H} \overline{\mathbf{X}}\right.
$$

where $\alpha^{(k+1)}$ is an adaptive regularization parameter, defined such that:

$$
\alpha^{(k+1)}=\frac{\left\|\overline{\mathbf{X}}-\overline{\mathbf{H}} \overline{\mathbf{F}}_{\mathbf{m}}^{(k)}\right\|_{2}^{2}}{\left\|\overline{\mathbf{W}}^{(k)^{1 / 2}} \overline{\mathbf{L}} \overline{\mathbf{F}}_{\mathbf{m}}^{(k)}\right\|_{2}^{2}}
$$

Practically, the adaptive regularization parameter automatically adjusts the amount of regularization throughout the iterative process, since its current value depends on the regularized solution computed at iteration $k$. This actually constitutes the definite advantage of the proposed multiplicative regularization over its additive counterpart, because updating the adaptive regularization parameter is almost costless.

\subsection{Choice of the initial solution and stopping criterion}

As any iterative procedure, the proposed IRLS algorithm requires the definition of a good initial guess and a reliable stopping criterion. 


\subsubsection{Choice of the initial solution}

The choice of the initial solution is essential for a successful reconstruction. This choice is all the more crucial that the functional to minimize is generally non-convex. Actually, the initial solution has to satisfy several prerequisites to lead to relevant reconstructions. More precisely, it could be defined as a coarse solution of the minimization problem, easy to compute, but sufficiently close to the actual solution to ensure the convergence of the iterative process. Such requirements are fulfilled by a solution of the form:

$$
\overline{\mathbf{F}}_{\mathbf{m}}^{(0)}=\left(\overline{\mathbf{H}}^{H} \overline{\mathbf{H}}+\alpha^{(0)} \overline{\mathbf{L}}^{H} \overline{\mathbf{L}}^{-1} \overline{\mathbf{H}}^{H} \overline{\mathbf{X}}\right.
$$

where $\alpha^{(0)}$ is a rough estimate of the converged value of the adaptive regularization parameter.

In Ref. [28], it has been shown that $\alpha^{(0)}$ can be chosen from the following heuristic procedure:

1. Find estimates of the largest and the smallest singular values of $\mathbf{A}=$ $\left[\overline{\mathbf{H}} \overline{\mathbf{L}}^{-1}\right]^{H}\left[\overline{\mathbf{H}} \overline{\mathbf{L}}^{-1}\right]$, noted $\widehat{\sigma}_{1}$ and $\widehat{\sigma}_{n}$ respectively.

The estimate of the largest singular value is given by the upper bound of $\sigma_{1}$, namely [54]:

$$
\widehat{\sigma}_{1}(\mathbf{A})=\sqrt{\|\mathbf{A}\|_{\infty}\|\mathbf{A}\|_{1}}
$$

The estimation of the smallest singular value is obtained from $\widehat{\sigma}_{1}$ and an estimate $\widehat{\kappa}$ of the condition number of $\mathbf{A}$, namely:

$$
\widehat{\sigma}_{n}(\mathbf{A})=\frac{\widehat{\sigma}_{1}(\mathbf{A})}{\widehat{\kappa}(\mathbf{A})}
$$


2. Define a set $S_{\alpha_{0}}$ of possible values of $\alpha^{(0)} \in\left[\widehat{\sigma}_{n}, \widehat{\sigma}_{1}\right]$ using a constant logarithmic spacing to take into account the decrease of the singular values.

3. Choose $\alpha^{(0)}=\operatorname{median}\left(S_{\alpha_{0}}\right)$.

Some comments should be made on the estimation procedure described above. This heuristic selection procedure has been developed with performance in mind. Indeed, classical automatic selection procedures, like the Generalized Cross Validation [39] or the L-curve principle [40], require the computation of the SVD of the system matrix, which can be time consuming when dealing with large reconstruction problems. To bypass this potential bottleneck, we have sought to develop a procedure close to the classical ones but avoiding the calculation of the SVD of the system matrix. That is why, estimates of the largest and smallest singular value are only calculated in step 1. Actually, all the performance gain are related to this calculation. The second step is rather standard in the implementation of automatic selection procedures [see Ref. [55] for instance]. The last step defines the initial adaptive regularization parameter as the median of the set $S_{\alpha_{0}}$. It has been found experimentally that such an estimate allows generally obtaining a relevant initial solution for the iterative solver. However, because this estimation procedure is heuristic, it may sometimes fail to give a good starting point for the iterative process. In such a situation, it is always possible to choose $\alpha^{(0)}$ as the regularization parameter picked by the L-curve or any other automatic selection procedure. Incidentally, the computational efficiency of the overall procedure is affected in proportion to the size of the transfer functions matrix. 


\subsection{Choice of the stopping criterion}

Regarding the choice of the stopping criterion, a classical approach consists in stopping the iterative process when the relative variation of a certain quantity is less or equal to some tolerance. Actually, the proposed iterative procedure offers a natural definition of the stopping criterion, based on the relative variation $\delta$ of the adaptive regularization parameter. By definition, this convergence indicator is written:

$$
\delta=\frac{\left|\alpha^{(k+1)}-\alpha^{(k)}\right|}{\alpha^{(k)}} .
$$

Experimentally, it has been found that setting the tolerance to $10^{-8}$ allows

obtaining consistent reconstruction, while preserving the time-performance of the IRLS algorithm [2].

\subsection{Generic resolution algorithm}

A comprehensive overview of the proposed resolution algorithm is given in table 1.

\section{Numerical validation}

This numerical validation intends to investigate the practical interest of applying the proposed approach for solving force reconstruction problems.

\subsection{Description of the numerical test case}

The studied structure is a thin simply supported steel beam of length $1 \mathrm{~m}$ and cross-sectional area $3 \times 10^{-4} \mathrm{~m}^{2}$. The beam is excited by a point force of unit amplitude from $50 \mathrm{~Hz}$ to $500 \mathrm{~Hz}$ (frequency resolution: $1 \mathrm{~Hz}$ ). The coordinate of the point force, measured from the left end of the beam, 
Table 1: Generic resolution algorithm

Inputs: Global transfer functions matrix $\overline{\mathbf{H}}$, Global measured vibration field $\overline{\mathbf{X}}$, Global differentiation matrix $\overline{\mathbf{L}}$, Tolerance tol

Output: Reconstructed force vector $\overline{\mathbf{F}}_{\mathbf{m}}$

Initialization: Estimate $\alpha^{(0)}$

Compute $\overline{\mathbf{F}}_{\mathbf{m}}^{(0)}$ from Eq. (25)

Compute $\epsilon$ from the cumulative histogram of $\left|\overline{\mathbf{L}} \overline{\mathbf{F}}_{\mathbf{m}}^{(0)}\right|$

Initialize $\delta$ to 1

\section{Iteration:}

while $\delta>$ tol

Compute $\overline{\mathbf{W}}^{(k)}$ from Eqs. (20), (21) and (22)

Compute $\alpha^{(k+1)}$ from Eq. (24)

Compute $\overline{\mathbf{F}}_{\mathbf{m}}^{(k+1)}$ from Eq. (23)

Update $\delta$ using Eq. (28)

$k \leftarrow k+1$

end

$\operatorname{return} \overline{\mathbf{F}}_{\mathbf{m}} \leftarrow \overline{\mathbf{F}}_{\mathbf{m}}^{(k)}$ 
is $x_{0}=0.6 \mathrm{~m}$. Practically, this excitation configuration allows not only simulating a broadband excitation such as a hammer impact, but also studying the influence of the proposed regularization term, since the excitation field spatially exhibits a sparse distribution of excitation sources.

To synthesize experimental vibration data, the global exact vibration displacement field $\overline{\mathbf{X}}_{\text {exact }}$ is first computed from a FE mesh of the beam made up with 20 plane beam elements, assuming that only transverse motions are measurable. Then, the exact displacement field is corrupted by an additive Gaussian white noise with a signal-to-noise ratio (SNR) equal to $30 \mathrm{~dB}$. It should be added that a structural damping ratio of 0.01 has been introduced in the calculation to avoid infinite displacement amplitudes at resonance frequencies, whose the five first are given in Table 2.

Table 2: First five resonance frequencies

\begin{tabular}{c|c}
\hline ID & Value \\
\hline 1 & $23.52 \mathrm{~Hz}$ \\
2 & $94.11 \mathrm{~Hz}$ \\
3 & $211.75 \mathrm{~Hz}$ \\
4 & $376.45 \mathrm{~Hz}$ \\
5 & $588.21 \mathrm{~Hz}$ \\
\hline
\end{tabular}

Regarding finally the definition of the reconstruction model, the global transfer functions matrix $\overline{\mathbf{H}}$ is computed from a FE model of the plate with 
free boundary conditions, by supposing that only the transverse forces acting on the structure are of primary importance. The main interest in using such a numerical model is to allow the reconstruction of both external and reaction forces $[4,11]$.

\subsection{Reference force vector}

To measure the ability of the proposed space-frequency regularization in reconstructing relevant excitation fields, it is first necessary to define a reliable point of comparison. Such a reference force vector is derived from the global transfer functions matrix $\overline{\mathbf{H}}$ and the global exact displacement $\overline{\mathbf{X}}_{\text {exact }}$ by using the following simple relation:

$$
\overline{\mathbf{F}}_{\text {ref }}=\overline{\mathbf{H}}^{-1} \overline{\mathbf{X}}_{\text {exact }}
$$

The typology of the sources acting on the structure is presented in Fig. 1. More specifically, this figure proposes a spatial representation of the reference force vector at a resonance frequency of the structure $(211 \mathrm{~Hz})$, but also at a frequency lying outside resonance frequencies $(150 \mathrm{~Hz})$. It should be noted that the reference force vector exhibits, as expected, only point sources, namely the point force and the reaction forces at both ends of the beam.

For the sake of completeness, the exact displacement field at $150 \mathrm{~Hz}$ and $211 \mathrm{~Hz}$ is given in Fig. 2. This figure clearly show that the analysis displacement vibration field alone does not provide any information on the sought excitation field, which emphasizes the interest of developing an appropriate reconstruction strategy. 


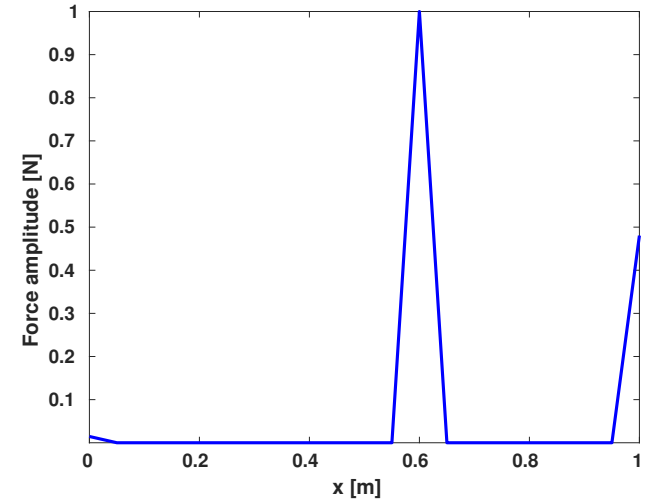

(a) $150 \mathrm{~Hz}$

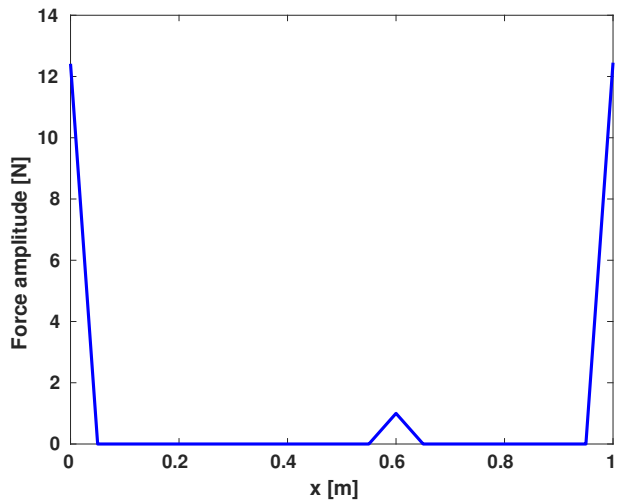

(b) $211 \mathrm{~Hz}$

Figure 1: Reference force vector $\overline{\mathbf{F}}_{\text {ref }}[:, j]$ (a) at a non-resonance frequency (150 Hz) and (b) at a resonance frequency $(211 \mathrm{~Hz})$

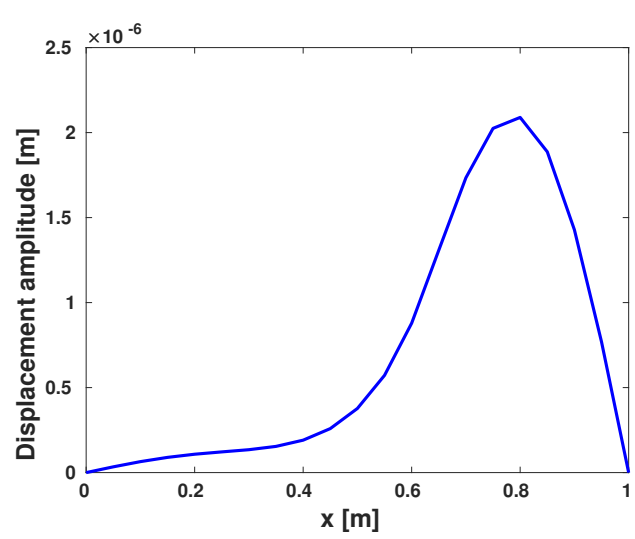

(a) $150 \mathrm{~Hz}$

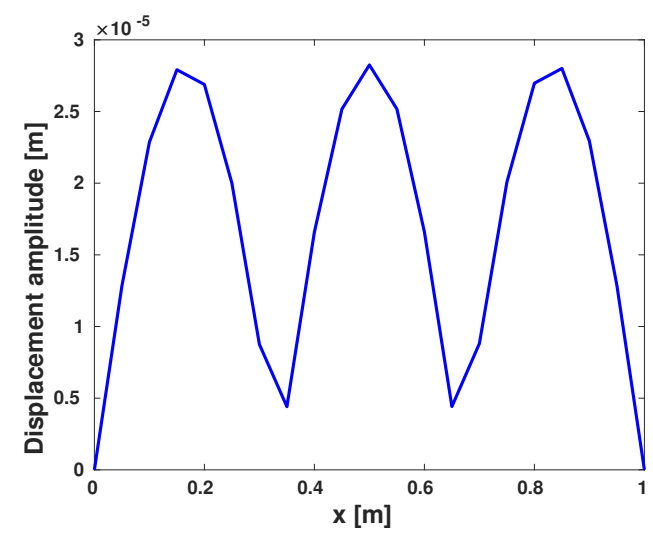

(b) $211 \mathrm{~Hz}$

Figure 2: Exact displacement vector $\overline{\mathbf{X}}_{\text {exact }}[:, j]$ (a) at a non-resonance frequency $(150 \mathrm{~Hz})$ and (b) at a resonance frequency $(211 \mathrm{~Hz})$ 


\subsection{Naive reconstruction}

When the displacement field is noisy, a naive approach is to solve the reconstruction problem in a least-squares sense, that is:

$$
\overline{\mathbf{F}}_{\text {naive }}=\overline{\mathbf{H}}^{+} \overline{\mathbf{X}}
$$

where $\overline{\mathbf{H}}^{+}$is the Moore-Penrose pseudoinverse of $\overline{\mathbf{H}}$.

Unfortunately, such a naive approach generally fails to locate and reconstruct the actual forces acting on a structure as illustrated in Figs. 3 and 4. It is especially noteworthy that the larger reconstruction errors of the point force spectrum are observed at resonance frequencies [see Fig. 4]. To justify this result, one has to keep in mind that for lightly damped structures the shape of the vibration response at resonance frequencies is close to that of the mode shapes. In this situation, the nearfield information is somewhat masked, which makes the reconstruction problem more involved. These preliminary results better explain the need for regularization methods and can serve as a basis to point out the main features of the proposed approach.

\subsection{Reconstruction from the space-frequency regularization}

To properly apply the proposed space-frequency regularization, one has to determine the parameters defining the regularization term. As already evoked in the introduction, consistent reconstructions can be obtained by properly exploiting the spatial and spectral characteristics of the force vector to identify. Regarding the spatial prior, one has to remind that the force vector is sparse [see Fig. 1]. Practically, this observation leads to define a parameter $q$ promoting the sparsity of the solution. On the other 


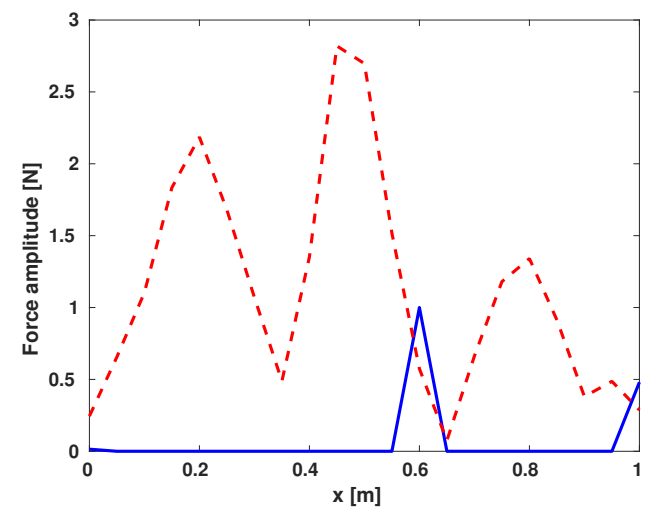

(a) $150 \mathrm{~Hz}$

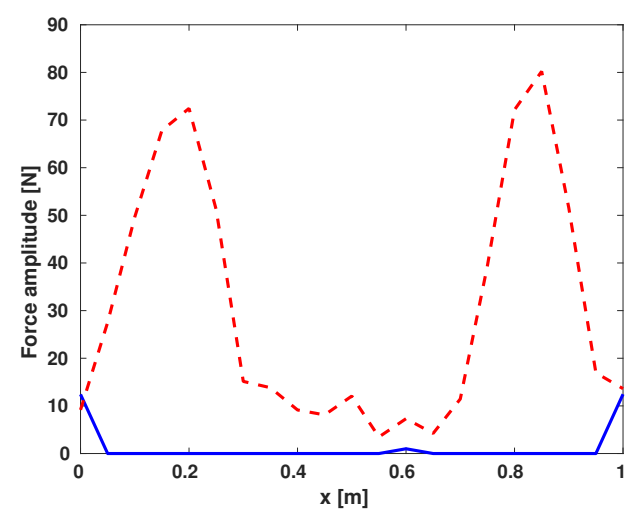

(b) $211 \mathrm{~Hz}$

Figure 3: Naive spatial reconstruction of the force vector $\overline{\mathbf{F}}_{\text {naive }}[j]$ (a) at a non-resonance frequency $(150 \mathrm{~Hz})$ and $(\mathrm{b})$ at a resonance frequency $(211 \mathrm{~Hz})-(-)$ Reference and (--) Naive reconstruction

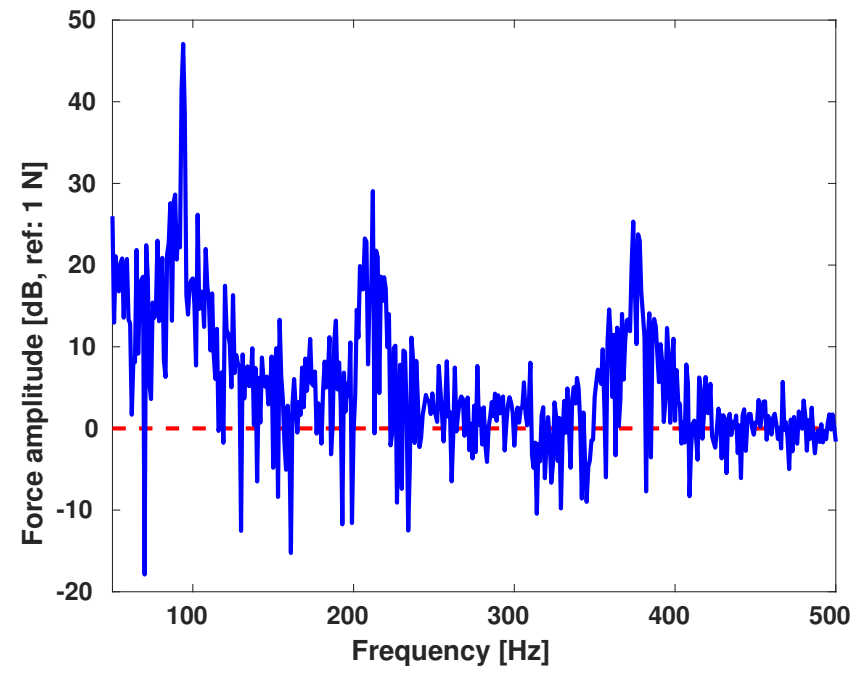

Figure 4: Spectrum of the reconstructed point force - (-) Naive reconstruction and (--) Reference 
hand, the point force excitation being broadband, the frequency prior has to reflect the continuity of the source signal. Following the recommendations given in section 3, one sets $(p, q)=(2,0.5)$ and $\overline{\mathbf{L}}=\mathbf{I}_{N M}$. To better highlight the advantage of the proposed regularization term [see Eq. 14], the proposed space-frequency $(\mathrm{SF})$ regularization is compared with the corresponding frequency-by-frequency $(\mathrm{FbF})$ regularization. The latter strategy is a particular case of the space-frequency approach, since it is obtained by defining $N$ separate frequency groups for a reconstruction point i. In other words, this regularization strategy is written [11]:

$$
\mathbf{F}_{\mathbf{m}}\left(f_{j}\right)=\underset{\mathbf{F}(j)}{\operatorname{argmin}}\left\|\mathbf{X}\left(f_{j}\right)-\mathbf{H}\left(f_{j}\right) \mathbf{F}\left(f_{j}\right)\right\|_{2}^{2} \cdot\left\|\mathbf{F}\left(f_{j}\right)\right\|_{q}^{q}, \quad \forall j=1, \ldots, N .
$$

The first step to assess the pertinence of the proposed SF regularization compared to the FbF strategy consists in appraising their ability in properly identifying the mechanical sources acting on the structure. The reconstructions proposed in Fig. 5 point out that both $\mathrm{FbF}$ and SF approaches provide a consistent identification of the reaction forces at boundaries. However, it is worth noting that the $\mathrm{FbF}$ regularization fails in localizing the point force at one of the resonance frequencies of the beam, which is not the case with the SF strategy [see Fig. 5b]. This observation is confirmed by the analysis of the spectrum of the point force reconstructed by the FbF or the SF approaches and presented in Fig. 6. Indeed, the force spectrum obtained using the FbF strategy exhibits large reconstruction errors around the resonance frequencies $(94 \mathrm{~Hz}, 211 \mathrm{~Hz}$ and $376 \mathrm{~Hz}$ in the frequency range of interest). On the contrary, the reconstruction error is limited to $1.6 \mathrm{~dB}$ at most over all the frequency range with the SF strategy. All these results tend to demonstrate that the proposed multiplicative regularization is well adapted to force 
reconstruction problems.

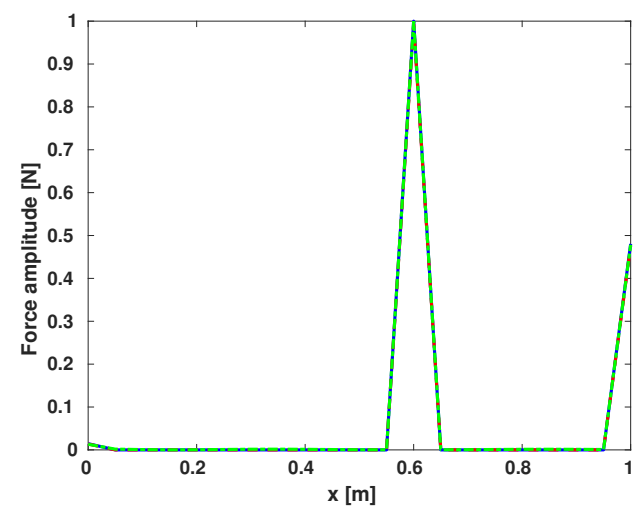

(a) $150 \mathrm{~Hz}$

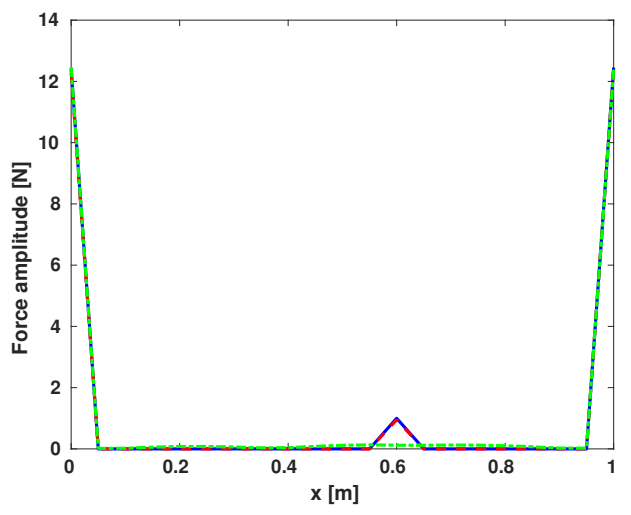

(b) $211 \mathrm{~Hz}$

Figure 5: Spatial reconstructions of the excitation field (a) at non-resonance frequency $(150 \mathrm{~Hz})$ and $(\mathrm{b})$ at a resonance frequency $(211 \mathrm{~Hz})$ of the beam - (-) Reference, (--) SF regularization and $(-\cdot-)$ FbF regularization

Finally, one has to notice that the proposed regularization strategy is well adapted to solve large reconstruction problem, since the calculation of a SVD, that is generally used to compute the optimal regularization parameter, is avoided. This is all the more interesting than the calculation of the SVD can be time-consuming or even impossible for large-scale systems on a personal computer.

\subsection{Influence of the choice of the norm parameters $p$ and $q$}

It can be argued that in real-life applications the proposed guidelines regarding the choice of the norm parameters $p$ and $q$ seem impractical, because the force distribution is unknown or there is no idea about the force to recover. We are prone to think that in real-life applications rough information 


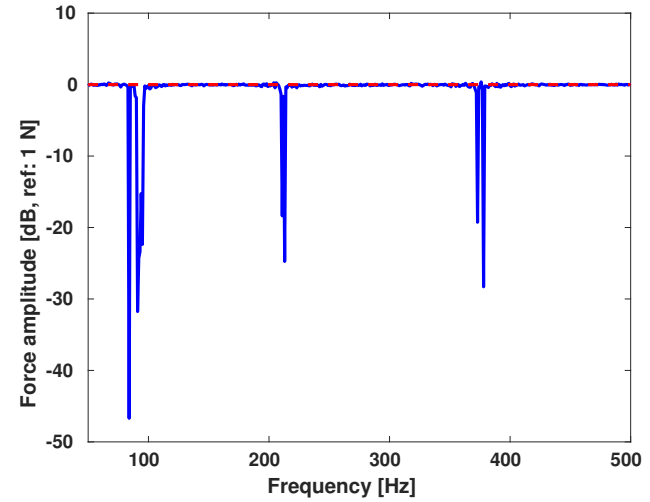

(a) FbF regularization

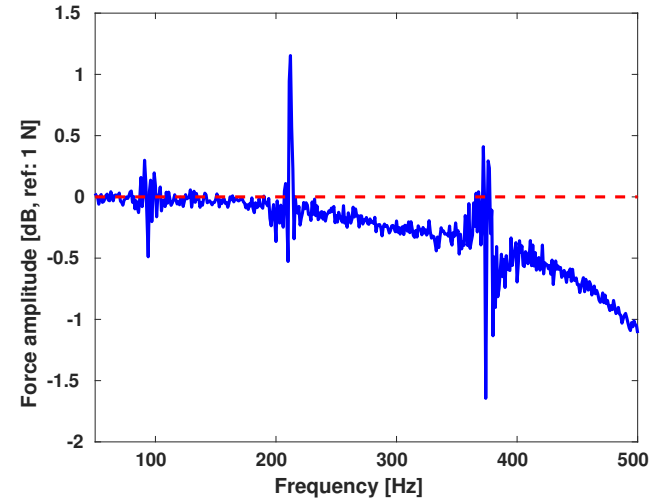

(b) SF regularization

Figure 6: Spectrum of the reconstructed point force - (-) Reconstructed force spectrum and (--) Reference force spectrum

about the force to recover is available. This information is provided by a careful analysis of the mechanical system. As a result, we think that a rough idea of the nature of the excitation signal and the type of the sources acting on the structure can be obtained, but this supposes that the user has some knowledge in mechanics.

Nevertheless, it is interesting to study the influence of an improper choice of $p$ and $q$ on the quality of the reconstructed solutions. To this end, two configurations are compared. The first one corresponds to the case where the the nature of the excitation signal is properly inferred, while the prior on the type of the source does not correspond to the actual spatial distribution. This situation typically arises when $(p, q)=(2,2)$. The second configuration corresponds to the situation for which the type of the source is properly determined, while the prior on the nature of the excitation signal does not 
match the actual one. This situation is encountered when $(p, q)=(1,0.5)$.

The analysis of the spatial reconstructions at $150 \mathrm{~Hz}$ presented in Fig. 7 tends to show that an error on the choice of $p$ seems less critical than an error on the choice of $q$. This observation is confirmed by the inspection of the spectrum of the reconstructed point force obtained from both configurations [see Fig. 8]. However, it should be noted that a poor choice of $p$ lowered the overall quality of the reconstructed solution even if $q$ is appropriately selected. This is particularly noticeable in Fig. 8b where the reconstruction error obtained for the second configuration with $p=1$ is on the whole greater than that obtained with $p=2$ [see Fig. 6b].

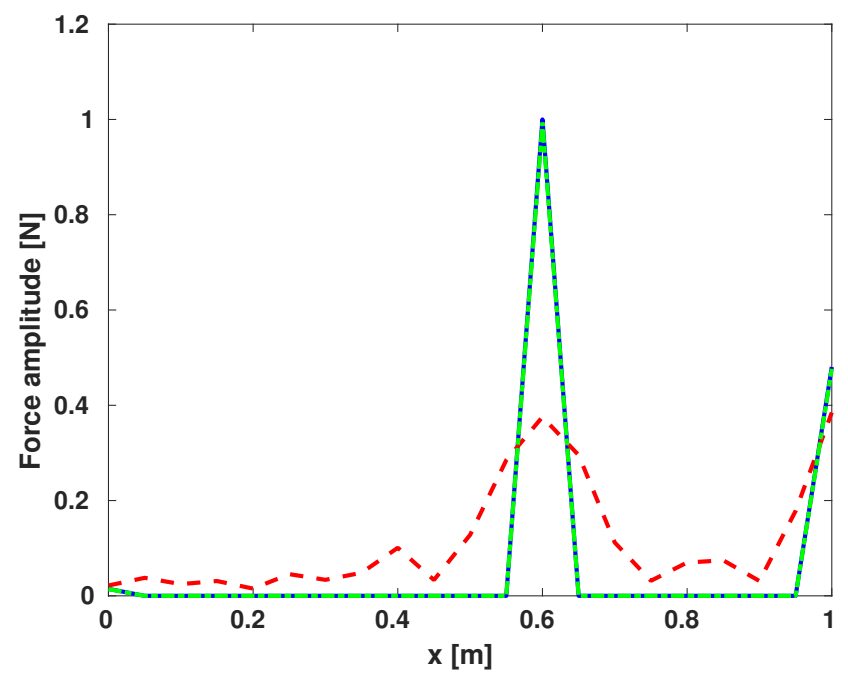

Figure 7: Spatial reconstructions of the excitation field at $150 \mathrm{~Hz}-(-)$ Reference, (--) Reconstructed excitation field for $(p, q)=(2,2)$ and $(-\cdot-)$ Reconstructed excitation field for $(p, q)=(1,0.5)$ 


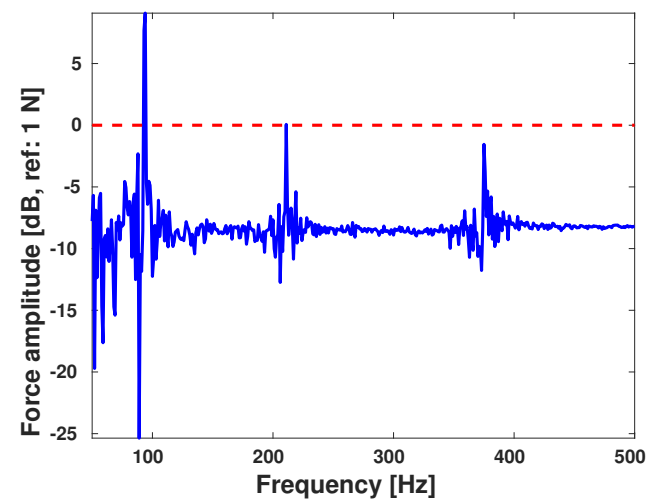

(a)

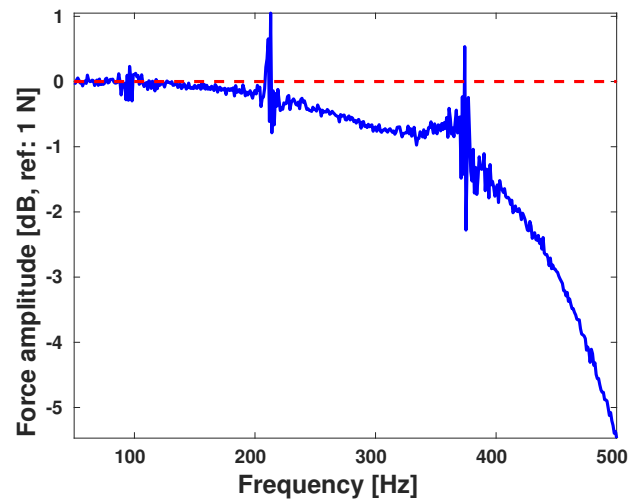

(b)

Figure 8: Spectrum of the reconstructed point force for (a) $(p, q)=(2,2)$ and (b) $(p, q)=$ $(1,0.5)$ - (-) Reconstructed force spectrum and (--) Reference force spectrum

\subsection{Influence of the noise on the performance of the resolution algorithm}

The proposed resolution algorithm being iterative, it could be interesting to determine how the noise corrupting the data affects its performances. To this end, $10^{3}$ samples of the measurement noise vector $\mathbf{N}$, supposed to be a Gaussian white noise, are drawn for each of two following SNR: 30 $\mathrm{dB}$ and $25 \mathrm{~dB}$. Then, the SF regularization is applied to each samples with $(p, q)=(2,0.5)$. This procedure allows quantifying the posterior uncertainty about the reconstructed solution by estimating the median and the $95 \%$ credible interval from the solution samples as well as monitoring the evolution of the number of iterations of the resolution algorithm.

As expected, Figs. 9 and 10 show that the posterior uncertainty about the reconstructed force vector is large at resonance frequencies and more generally increases as the SNR decreases, which is also associated to a loss 
of accuracy of the reconstruction. Regarding the number of iterations performed by the resolution algorithm to converge, the results are summarized in Table 3. It can be seen that the number of iterations increases as the SNR decreases, which is in line with our expectations. It is also worth noting that the related $95 \%$ credible interval around the median value is relatively sharp.

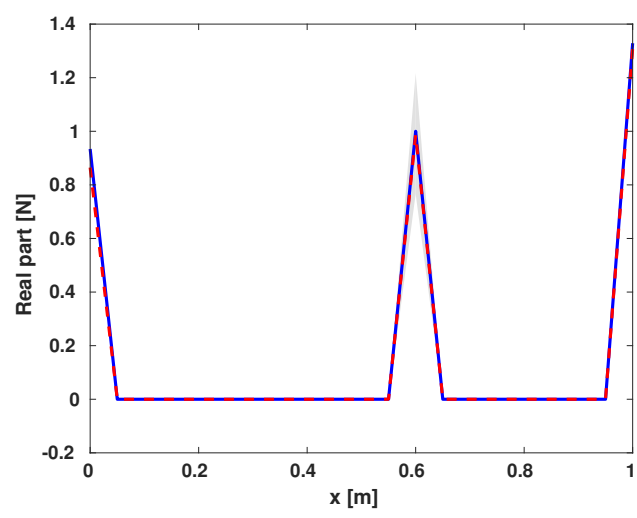

(a)

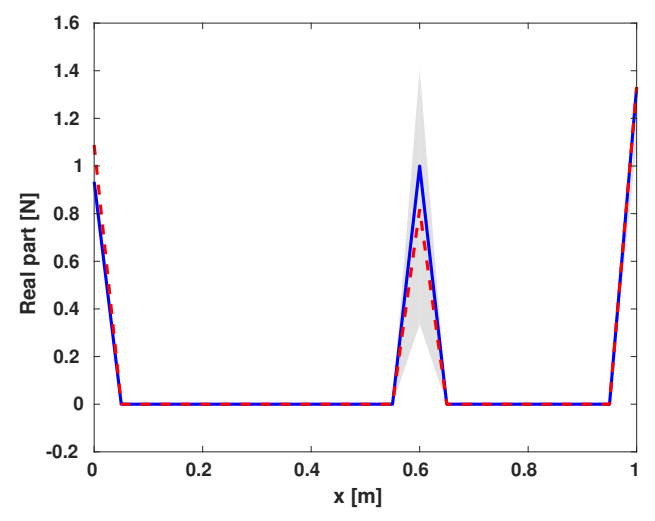

(b)

Figure 9: Spatial reconstructions of the excitation field at $211 \mathrm{~Hz}$ for a SNR equal to (a) $30 \mathrm{~dB}$ and (b) $25 \mathrm{~dB}$ - Real part of the reconstructed force vector - (-) Reference, (--) Median of the samples and $(\square) 95 \%$ credible interval

Table 3: Number of iteration with respect to the SNR

\begin{tabular}{c|cc}
\hline SNR & Median & $95 \%$ CI \\
\hline $30 \mathrm{~dB}$ & 20 & {$[19,23]$} \\
$25 \mathrm{~dB}$ & 28 & {$[25,31]$} \\
\hline
\end{tabular}




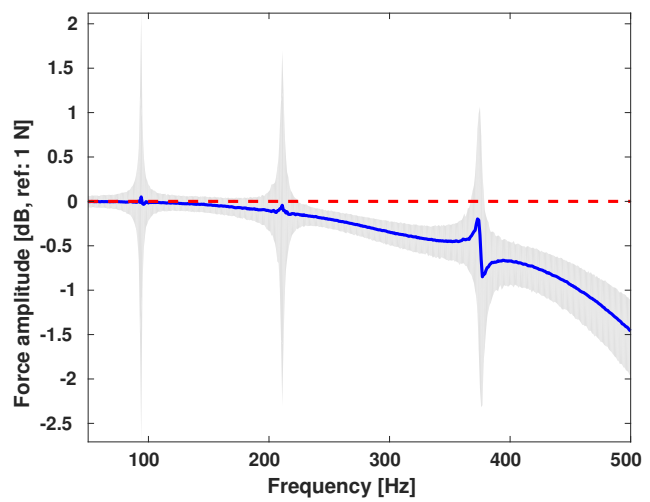

(a)

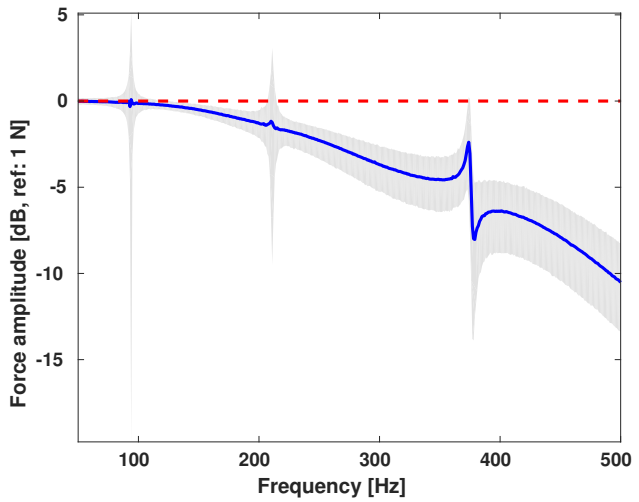

(b)

Figure 10: Spectrum of the reconstructed point force for a SNR equal to (a) $30 \mathrm{~dB}$ and (b) $25 \mathrm{~dB}$ - Real part of the reconstructed force vector - (-) Median of the samples, (--) Reference force spectrum and $(\square) 95 \%$ credible interval

\section{Experimental validation}

To confirm the conclusions drawn from the previous numerical experiment, the $\mathrm{SF}$ and $\mathrm{FbF}$ regularizations are applied to a real structure. The main objective of this experimental validation is to assess the performance of the proposed approach in operating conditions.

\subsection{Description of the experimental set-up}

The structure under test is a suspended (free) aluminum plate of $0.6 \mathrm{~m}$ in length, $0.4 \mathrm{~m}$ in width and $5 \mathrm{~mm}$ in thickness [see Fig. 11a]. The plate is excited at $\left(x_{0}, y_{0}\right)=(0.405 \mathrm{~m}, 0.255 \mathrm{~m})$ by a shaker fed by a white noise signal and equipped with a force sensor [see Fig. 11b].

Measurements of the vibration field were carried out with a scanning laser 


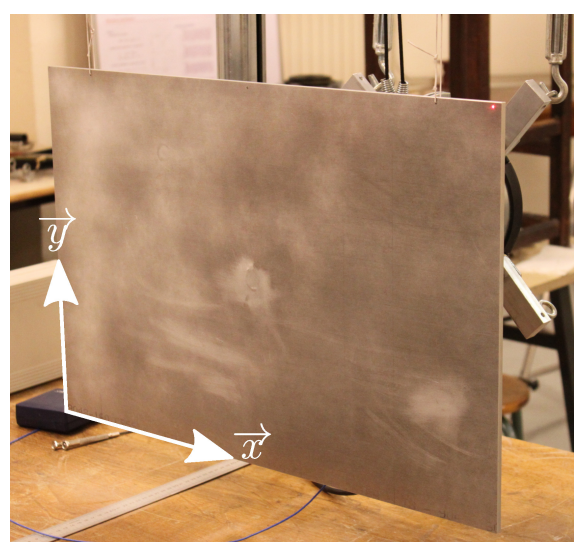

(a)

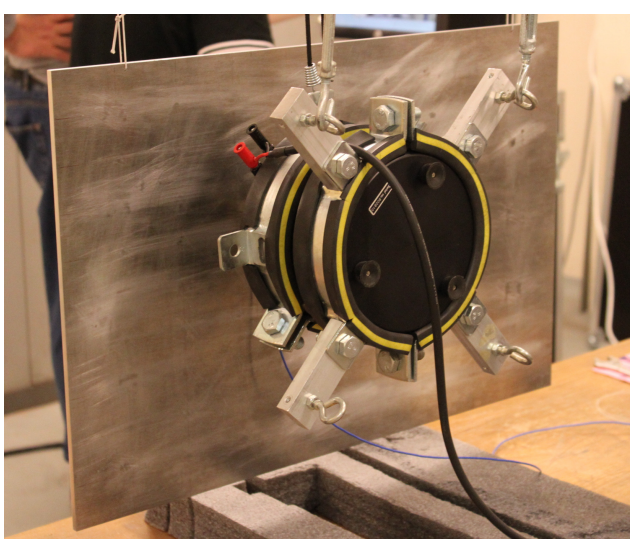

(b)

Figure 11: Experimental set-up - (a) Suspended plate and (b) Excitation device

vibrometer on a grid of $35 \times 29$ points along $\mathrm{x}$ and $\mathrm{y}$ directions respectively using the force signal as phase reference. Regarding the FE mesh used to model the dynamic behavior of the plate and reconstruct the excitation field, it has been designed to perfectly match the measurement mesh. As a result, it consists of 952 shell elements, making the model theoretically valid up to $4500 \mathrm{~Hz}$. Then, the corresponding FE model with free boundary conditions has been used to compute the transfer functions matrix $\mathbf{H}$, considering the bending motions as the only available data. Finally, it is worth mentioning that the FE model has not been updated in terms of Young modulus and density and that a global structural damping, estimated from the modal damping ratios obtained from the measured FRFs, has been used in the present experimental validation. 


\subsection{Reconstruction of the excitation field}

In the numerical validation, the proposed SF formulation has been compared with the related $\mathrm{FbF}$ regularization. A similar analysis process is proposed in this section. The analysis of the experimental set-up implies that the plate is only excited by a broadband point force. Consequently, it is necessary to define a regularization promoting the spatial sparsity of the excitation field as well as the continuity of the force spectrum. From the guidelines given in section 3.1, the tuning parameters of the SF regularization term are sets to $(p, q)=(2,0.5)$.

Figure 12 presents a comparison of the force spectrum identified at $\left(\widehat{x_{0}}, \widehat{y_{0}}\right)=$ (0.404 $\mathrm{m}, 0.256 \mathrm{~m})$ by the $\mathrm{SF}$ and FbF regularizations with the reference signal measured by the force sensor between $150 \mathrm{~Hz}$ and $750 \mathrm{~Hz}$ (frequency resolution: $5 \mathrm{~Hz}$ ). Contrary to what has been observed in the numerical validation, the $\mathrm{SF}$ and $\mathrm{FbF}$ regularizations provide similar results on the whole, but exhibit discrepancies at some resonance frequencies of the plate. To further compare the $\mathrm{SF}$ and $\mathrm{FbF}$ regularizations, it is interesting to see whether the reconstructed force spectrum far from the excitation point is close to $0 \mathrm{~N}$. This expected result is confirmed in Fig. 13, where the reconstructed force spectrum at $(x, y)=(0.109 \mathrm{~m}, 0.075 \mathrm{~m})$ obtained from both regularization strategies are indeed close to $0 \mathrm{~N}$. Although these results seem consistent with our expectations, it remains to verify the behavior of the SF and $\mathrm{FbF}$ approaches in the space domain. Indeed, the excitation field being reconstructed on a grid of $35 \times 29$ points, corresponding to the measurement

mesh, it is possible to study its spatial distribution. One can observe in 
Fig. 14 that both regularization techniques allows identifying relevant force distributions, since the point force is properly localized. More specifically, they perform equally well at non-resonance frequency (here $230 \mathrm{~Hz}$ ), insofar as the point force amplitude is respectively estimated to $0.030 \mathrm{~N}$ and 0.036 $\mathrm{N}$ by the $\mathrm{SF}$ and FbF regularizations, while the measured amplitude is equal to 0.029 N. Furthermore, as already observed in the numerical validation and in Fig. 12, the main discrepancies can arises around the resonance frequencies. For instance, Fig. 14 points out that at $290 \mathrm{~Hz}$, the SF and FbF regularizations behave rather differently, since the SF leads to a consistent reconstructed solution (identified: $0.015 \mathrm{~N}$, actual: $0.014 \mathrm{~N}$ ). On the contrary, the FbF strategy fails in properly estimating the point force amplitude (identified: $0.002 \mathrm{~N}$ ). However, except this very local difference, both techniques provide similar results on whole frequency range [see Fig. 12]. In the end, the present experimental study shows that, in a particular real-world application, the SF multiplicative regularization leads to a reconstruction similar to that obtain with the related $\mathrm{FbF}$ multiplicative regularization. However, this observation is finally reassuring here, because the solution provided by the FbF strategy is already in good agreement with the reference one.

\section{Extension to time domain applications}

The proposed space-frequency regularization strategy can be easily adapted to derive a space-time regularization approach. To this end, only the global reconstruction model has to be modified. If the structure is time-invariant and initially at rest, the displacement field $\mathbf{x}_{k}$ at instant $t_{k}$ is obtained from 


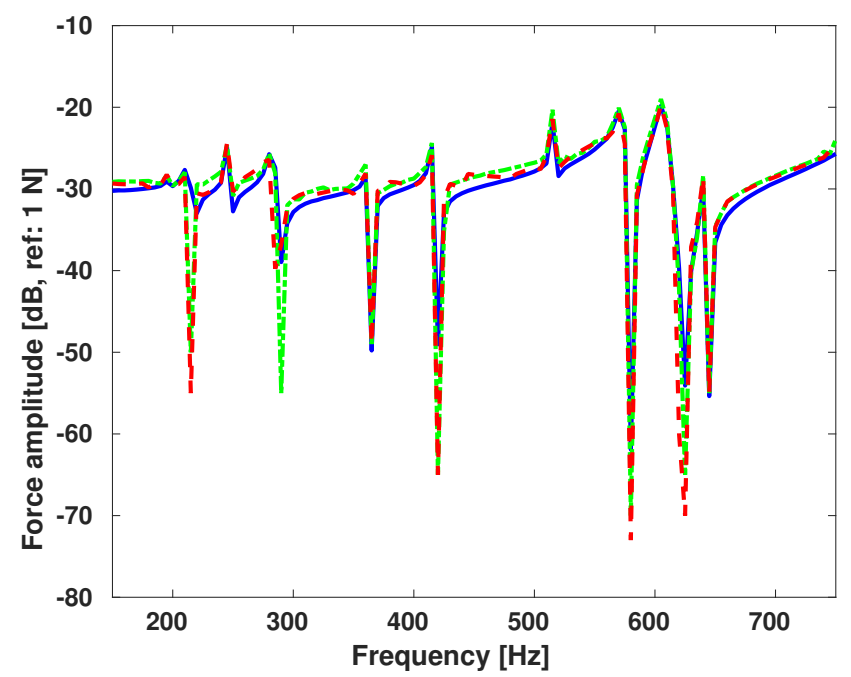

Figure 12: Reconstruction of the force spectrum at identified excitation point $-(-)$ Reference measured by the force sensor, (--) SF regularization and (-.-) FbF regularization

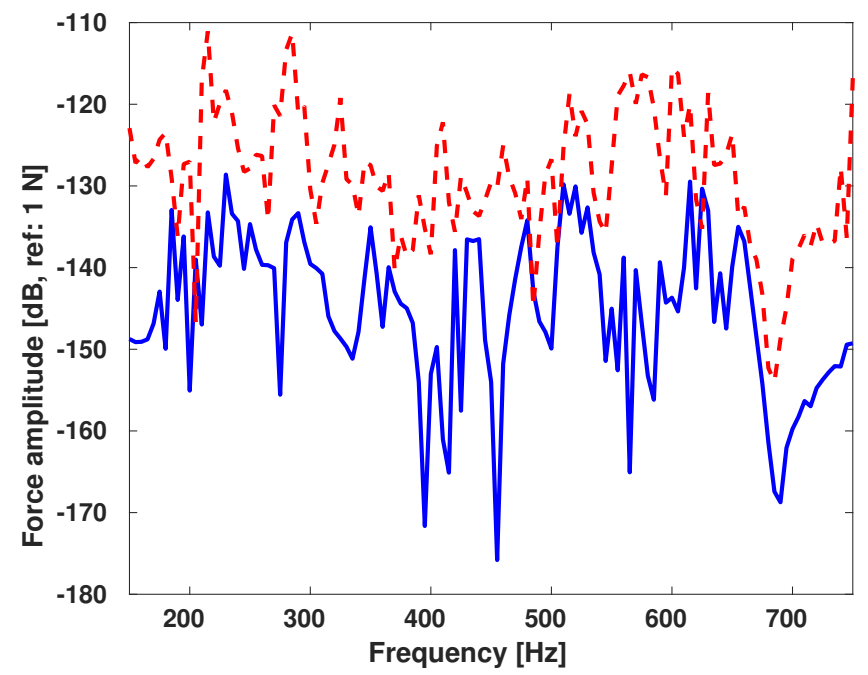

Figure 13: Reconstruction of the force spectrum far from the identified excitation point (-) SF regularization and(--) FbF regularization 


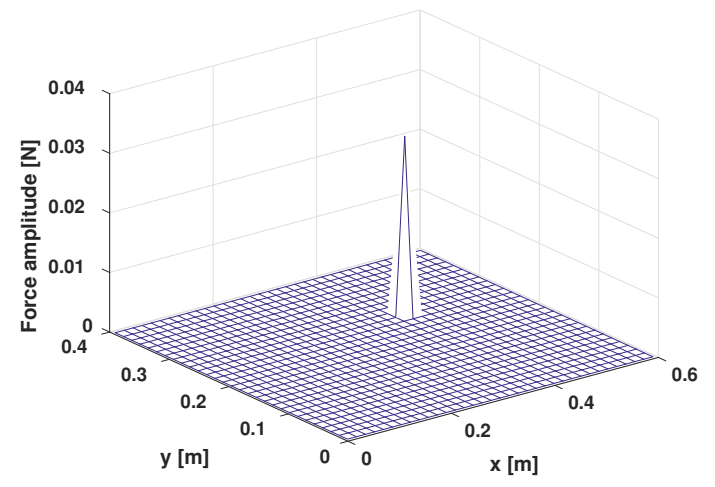

(a) $230 \mathrm{~Hz}-\mathrm{SF}$

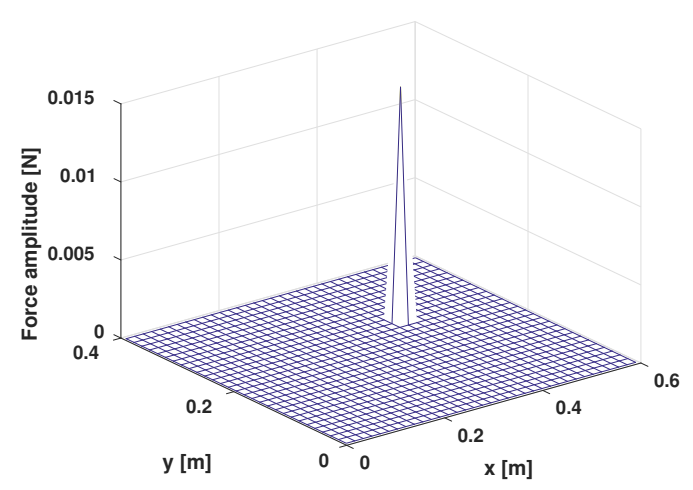

(c) $290 \mathrm{~Hz}-\mathrm{SF}$

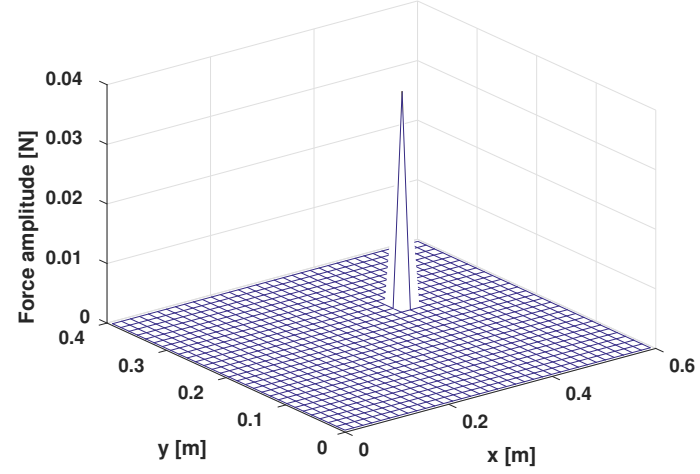

(b) $230 \mathrm{~Hz}-\mathrm{FbF}$

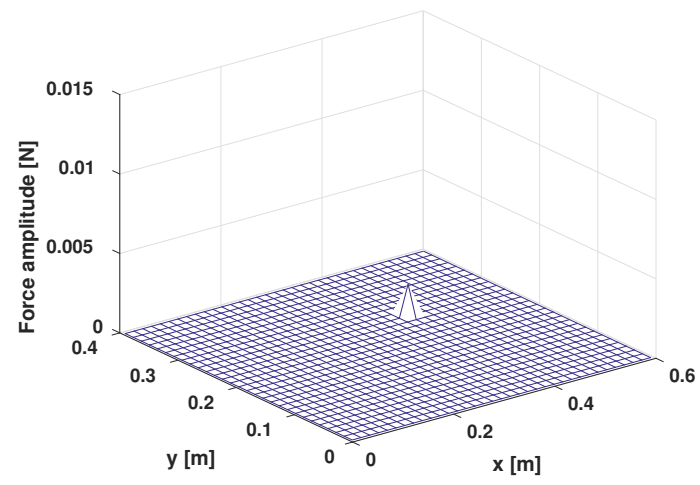

(d) $290 \mathrm{~Hz}-\mathrm{FbF}$

Figure 14: Spatial reconstructions of the excitation field at non-resonance frequency (230 $\mathrm{Hz})$ and around a resonance frequency of the plate $(290 \mathrm{~Hz})-(\mathrm{a}, \mathrm{c}) \mathrm{SF}$ regularization and (b, d) FbF regularization 
the discretized Duhamel's integral:

$$
\mathbf{x}_{k}=\Delta t \sum_{i=0}^{k} \mathbf{h}_{k-i} \mathbf{f}_{i}+\mathbf{n}_{k}
$$

where $\Delta t=t_{k+1}-t_{k}$ is the constant time step and $\mathbf{h}_{k}$ and $\mathbf{n}_{k}$ are respectively the impulse response matrix and the noise vector at instant $t_{k}$.

The previous relation can be recast under the following matrix form:

$$
\overline{\mathrm{X}}=\overline{\mathbf{H}} \overline{\mathbf{F}}+\overline{\mathbf{N}}
$$

with:

$$
\overline{\mathbf{H}}=\left[\begin{array}{cccc}
\mathbf{h}_{0} & \mathbf{0} & \cdots & \mathbf{0} \\
\mathbf{h}_{1} & \mathbf{h}_{0} & \ddots & \vdots \\
\vdots & \vdots & \ddots & \mathbf{0} \\
\mathbf{h}_{N_{t}} & \mathbf{h}_{N_{t}-1} & \cdots & \mathbf{h}_{0}
\end{array}\right], \quad \overline{\mathbf{X}}=\left[\begin{array}{c}
\mathbf{x}_{0} \\
\mathbf{x}_{1} \\
\vdots \\
\mathbf{x}_{N_{t}}
\end{array}\right], \overline{\mathbf{F}}=\left[\begin{array}{c}
\mathbf{f}_{0} \\
\mathbf{f}_{1} \\
\vdots \\
\mathbf{f}_{N_{t}}
\end{array}\right], \quad \overline{\mathbf{N}}=\left[\begin{array}{c}
\mathbf{n}_{0} \\
\mathbf{n}_{1} \\
\vdots \\
\mathbf{n}_{N_{t}}
\end{array}\right]
$$

where $N_{t}$ is the total number of time steps.

Consequently, using the previous mechanical model, the regularization procedure described in sections 3 and 4 can be directly implemented for time domain applications. It is also worth mentioning that the such a space-time regularization is a natural strategy for time domain applications, for which the reconstruction problem is classically established from a convolution model $[13,56]$. We are prone to think that the extension and application of the proposed regularization strategy to force reconstruction problems in time domain is very promising, because of its ability to allow dissociate prior 
information on the spatial distribution and the time history of the force signals.

\section{Conclusion}

In the present study, the initial motivation was to propose a formulation of the force reconstruction problem able to fully exploit information available a priori on the type of the sources and the nature of the excitation signals. To this end, a space-frequency multiplicative regularization has been introduced. This formulation is highly flexible, since it allows dealing with various force distributions and various excitation signals within a unique framework. Practically, the regularization problem is solved from an adapted IRLS algorithm. The proposed experimental application tends to show that the SF multiplicative regularization gives results similar to those obtained with the related FbF multiplicative regularization. This apparently disappointing observation is actually reassuring, since, in the present case, the force signal identified by the FbF strategy is already in good agreement with the reference one. The potential benefit of the proposed SF strategy has been better highlight in the numerical validation, since it allows obtaining consistent reconstructions even at structural resonance frequencies, where the FbF approach generally fails. These proposed numerical and experimental applications consequently indicate that the $\mathrm{SF}$ regularization performs better than or, at least, similarly to the FbF regularization. Finally, a direct and natural promising extension of the proposed regularization has been introduced for time domain application. Its implementation and its performances will be the subject of a future publication. 


\section{Appendix A. Calculation of the weighting coefficients}

The purpose of this appendix is to detail the calculation of the weighting coefficients $W_{i, j}^{f(k)}$ and $W_{i}^{s(k)}$. To this end, let us define $\overline{\mathbf{Y}}=\overline{\mathbf{L}} \overline{\mathbf{F}}_{\mathbf{m}}^{(k)}$ and $\overline{\mathbf{Y}}[i,:]=\left(\overline{\mathbf{L}} \overline{\mathbf{F}}_{\mathbf{m}}^{(k)}\right)[i,:]$. From these definitions, the weighting coefficients are determined as follows:

$$
\begin{aligned}
\|\overline{\mathbf{Y}}[i,:]\|_{p}^{p} & =\sum_{j=1}^{N}\left|Y_{i}\left(f_{j}\right)\right|^{p} \\
& =\sum_{j=1}^{N}\left|Y_{i}\left(f_{j}\right)\right|^{p-2}\left|Y_{i}\left(f_{j}\right)\right|^{2} \\
& =\sum_{j=1}^{N} W_{i, j}^{f(k)}\left|Y_{i}\left(f_{j}\right)\right|^{2} \\
& =\left\|\mathbf{W}_{\mathbf{i}}^{\mathbf{f}(k)^{1 / 2}} \overline{\mathbf{Y}}[i,:]\right\|_{2}^{2},
\end{aligned}
$$

where $\mathbf{W}_{\mathbf{i}}^{\mathbf{f}(k)}=\operatorname{diag}\left(W_{i, 1}^{f(k)}, \ldots, W_{i, N}^{f(k)}\right)$.

The previous equation clearly shows that the weighting coefficients $W_{i, j}^{f(k)}$ are theoretically defined by:

$$
W_{i, j}^{f(k)}=\left|Y_{i}\left(f_{j}\right)\right|^{p-2} .
$$

However, to avoid infinite weights when $\left|Y_{i}\left(f_{j}\right)\right| \rightarrow 0$ and $p<2$, a damping parameter $\epsilon_{f}$ must be introduced.

The weighting coefficients $W_{i}^{s(k)}$ are obtained in a similar fashion, that 
is:

$$
\begin{aligned}
& \|\overline{\mathbf{Y}}\|_{p, q}^{q}=\sum_{i=1}^{M}\left(\|\overline{\mathbf{Y}}[i,:]\|_{p}^{p}\right)^{\frac{q}{p}} \\
& =\sum_{i=1}^{M}\left(\left\|\mathbf{W}_{\mathbf{i}}^{\mathbf{f}(k)^{1 / 2}} \overline{\mathbf{Y}}[i,:]\right\|_{2}^{2}\right)^{\frac{q}{p}} \\
& =\sum_{i=1}^{M}\left(\left\|\mathbf{W}_{\mathbf{i}}^{\mathbf{f}(k)^{1 / 2}} \overline{\mathbf{Y}}[i,:]\right\|_{2}^{2}\right)^{\frac{q}{p}-1}\left\|\mathbf{W}_{\mathbf{i}}^{\mathbf{f}(k)^{1 / 2}} \overline{\mathbf{Y}}[i,:]\right\|_{2}^{2} \\
& =\sum_{i=1}^{M} W_{i}^{s(k)}\left\|\mathbf{W}_{\mathbf{i}}^{\mathbf{f}(k)^{1 / 2}} \overline{\mathbf{Y}}[i,:]\right\|_{2}^{2} \\
& =\left\|\overline{\mathbf{W}}^{(k)^{1 / 2}} \overline{\mathbf{Y}}\right\|_{2}^{2} \text {. }
\end{aligned}
$$

From Eq. (A.3), the weighting coefficients $W_{i}^{s(k)}$ are simply given by:

$$
W_{i}^{s(k)}=\left(\left\|\mathbf{W}_{\mathbf{i}}^{\mathbf{f}(k)^{1 / 2}} \overline{\mathbf{Y}}[i,:]\right\|_{2}^{2}\right)^{\frac{q}{p}-1}
$$

As stated previously, a damping parameter $\epsilon_{s}$ should be introduced in order to avoid infinite weights when $\left\|\mathbf{W}_{\mathbf{i}}^{\mathbf{f}(k)^{1 / 2}} \overline{\mathbf{Y}}[i,:]\right\|_{2} \rightarrow 0$ and $q<p$. For the sake of simplicity, the damping parameter is chosen such that $\epsilon_{f}=\epsilon_{s}=\epsilon$, where $\epsilon$ is calculated from the cumulative histogram of $\left|\overline{\mathbf{L}} \overline{\mathbf{F}}_{\mathbf{m}}^{(0)}\right|$ [see section 4.1].

Finally, Eq. (A.3) allows also demonstrating that the coefficients of the global weighting matrix $\overline{\mathbf{W}}^{(k)}$ are written:

$$
\overline{\mathrm{W}}_{I}^{(k)}=W_{i}^{s(k)} W_{i, j}^{f(k)}
$$

where $I=j+N(i-1)$. 


\section{References}

[1] A. N. Tikhonov. Regularization of incorrectly posed problems. Soviet Mathematics, 4:1624-1627, 1963.

[2] M. Aucejo. Structural source identification using a generalized Tikhonov regularization. Journal of Sound and Vibration, 333(22):5693-5707, 2014.

[3] S. Boyd and L. Vandenberghe. Convex optimization. Cambridge University Press, 2004.

[4] C. Renzi, C. Pezerat, and J.-L. Guyader. Vibratory source identification by using the finite element model of a subdomain of a flexural beam. Journal of Sound and Vibration, 332:545-562, 2013.

[5] R. Tibshirani. Regression shrinkage and selection via the lasso. Journal of the Royal Statistical Society, 58 (1):267-288, 1996.

[6] H. Fu, K. Ng, M. Nikolova, and J. L . Barlow. Efficient minimization methods of mixed $l_{2}-l_{1}$ and $l_{1}-l_{1}$ norms for image restoration. SIAM Journal on Scientific Computing, 27 (6):1881-1902, 2006.

[7] R. Chartrand and V. Stavena. Nonconvex regularization for image segmentation. In Proccedings of International Conference on Image Processing, Computer Vision and Pattern Recognition (IPCV) 200\%, Las Vegas, USA, 2007.

[8] M. Grasmair. Non-convex sparse regularization. Journal of Mathematical Analysis and Applications, 365 (1):19-28, 2010. 
[9] N. Chu, A. Mohammad-Djafari, and J. Picheral. Robust bayesian superresolution approach via sparsity enforcing a priori for near-field aeroacoustic source imaging. Journal of Sound and Vibrations, 332 (18):43694389, 2013.

[10] M. Aucejo and O. De Smet. Bayesian structural source identification using local generalized gaussian priors. In Internoise 2013, Innsbruck, Austria, 2013.

[11] M. Aucejo and O. De Smet. Bayesian source identification using local priors. Mechanical Systems and Signal Processing, 66-67:120-136, 2016.

[12] A. Rezayat, V. Nassiri, B. De Pauw, J. Ertveldt, S. Vanlanduit, and P. Guillaume. Identification of dynamic forces using group-sparsity in frequency domain. Mechanical Systems and Signal Processing, 7071:756-768, 2016.

[13] E. Jacquelin, A. Bennani, and P. Hamelin. Force reconstruction: analysis and regularization of a deconvolution. Journal of Sound and Vibration, 265:81-107, 2003.

[14] Y.-M Mao, X.-L. Guo, and Y. Zhao. Experimental study of hammer impact identification on a steel cantilever beam. Experimental Techniques, $34(3): 82-85,2010$.

[15] T. S. Jang, H. Baek, S. L. Han, and T. Kinoshita. Indirect measurement of the impulsive load to a nonlinear system from dynamic responses: Inverse problem formulation. Mechanical Systems and Signal Processing, 24:1665-1681, 2010. 
[16] E. Turco. Tools for the numerical solution of inverse problems in structural mechanics: review and research perspectives. European Journal of Environmental and Civil Engineering, 21 (5):509-554, 2017.

[17] R. T. Jones, J. S. Sirkis, and E. J. Friebele. Detection of impact location and magnitude for isotropic plates using neural networks. Journal of Intelligent Material Systems and Structures, 8:90-99, 1997.

[18] J. Park, S. Ha, and F.-K. Chang. Monitoring Impact Events Using a System-Identification Method. The AIAA Journal, 47(9):2011-2021, 2009.

[19] E. Lourens, E. Reynders, G. De Roeck, G. Degrande, and G. Lombaert. An augmented kalman filter for force identification in structural dynamics. Mechanical Systems and Signal Processing, 27:446-460, 2012.

[20] D. Ginsberg and C. P. Fritzen. New approach for impact detection by finding sparse solution. In Proceedings of ISMA 2014, 2014.

[21] A. Rezayat, V. Nassiri, S. Vanlanduit, and P. Guillaume. Force identification using mixed and penalized optimization techniques. In Proceedings of ISMA 2014, 2014.

[22] E. Turco. A strategy to identify excitating forces acting on structures. International Journal for Numerial Methods in Engineering, 64:14831508, 2005.

[23] M. Kowalski. Sparse regression using mixed norms. Applied and Computational Harmonic Analysis, 27:303-324, 2009. 
[24] A. Gramfort, M. Kowalski, and M. Hamalainen. Mixed-norm estimates for the $\mathrm{m} /$ eeg inverse problem using accelerated gradient methods. Physics in Medecine and Biology, 57:1937-1961, 2012.

[25] C. Zheng, G. Li, Y. Liu, and X. Wang. Subspace weighted $\ell_{2,1}$ minimization for sparse signal recovery. EURASIP Journal on Advances in Signal Processing 2012, 2012:98, 2012.

[26] Y. Wang, J. Wang, and Z. Xu. On recovery of block-sparse signals via mixed $\ell_{2} / \ell_{q}(0<q \leq 1)$ norm minimization. EURASIP Journal on Advances in Signal Processing 2013, 2013:76, 2013.

[27] P. M. van den Berg, A. Abubakar, and J. T. Fokkema. Multiplicative regularization for constrast profile inversion. Radio Science, 38:80228031, 2003.

[28] M. Aucejo and O. De Smet. A multiplicative regularization for force reconstruction. Mechanical Systems and Signal Processing, 85:730-745, 2017.

[29] M. H. A. Janssens and J. W. Verheij. A pseudo-forces methodology to be used in characterization of structure-borne sound sources. Applied Acoustics, 61:285-308, 2000.

[30] P. Guillaume, E. Parloo, P. Verboven, and G. De Sitter. An inverse method for the identification of localized excitation sources. In Proceedings of the 20th International Modal Analysis Conference, Los Angeles, USA, 2002. 
[31] E. Parloo, P. Verbiven, P. Guillaume, and M. Van Overmeire. Force identification by means of in-operation modal models. Journal of Sound and Vibration, 262 (1):161-173, 2003.

[32] B. J. Dobson and E. Rider. A review of the indirect calculation of excitation forces from measured structural response data. Journal of Mechanical Engineering Science, 204:69-75, 1990.

[33] Q. Leclere, C. Pezerat, B. Laulagnet, and L. Polac. Indirect measurement of main bearing loads in an operating diesel engine. Journal of Sound and Vibration, 286 (1-2):341-361, 2005.

[34] M. Green. Statistics of Images, the TV Algorithm of Rudin-OsherFatemi for Image Denoising and an Improved Denoising Algorithm. Technical report, UCLA, 2002.

[35] M. Nikolova. A variational approach to remove outliers and impulse noise. Journal of Mathematical Imaging and Vision, 20 (1-2):99-120, 2004.

[36] T. Le, R. Chartrand, and T. J. Asaki. A variational approach to reconstructing images corrupted by poisson noise. Journal of Mathematical Imaging and Vision, 27:257-263, 2007.

[37] A. Chambolle. Total variation minimization and a class of binary mrf models. Lecture Notes in Computer Science, 3757/2005:136-152, 2005.

[38] M. Hintermuller and T. Wu. Nonconvex TV ${ }^{\mathrm{q}}$-models in image restoration: Analysis and a trust-region regularization based superlineraly con- 
vergennt solver. Technical report, Institute of Mathematics ans Scientific Computing, University of Graz, 2011.

[39] G. H. Golub, M. Heath, and G. Wahba. Generalized cross-validation as a method for choosing a good ridge parameter. Technometrics, 21 (2):215-223, 1979.

[40] P. C. Hansen. Rank-Deficient and Discrete Ill-Posed Problems: Numerical Aspects of Linear Inversion. SIAM, 1998.

[41] A. Abubakar and P. M. van den Berg. Two - and three-dimensional algorithms for microwave imaging and inverse scattering. Journal of Electromagnetic Waves and Applications, 17:209-231, 2003.

[42] A. Abubakar, P. M. van den Berg, T. M. Habashy, and H. Braunish. A multiplicative regularization approach for deblurring problems. IEEE Transactions on Image Processing, 13:1524-1532, 2004.

[43] A. Abubakar, P. M. van den Berg, and T. M. Habashy. An integral equation approach for 2.5-dimensional forward and inverse electromagnetic scattering. Geophysics Journal International, 165:744-762, 2006.

[44] L. L. Li and B. Jafarpour. Effective solution of nonlinear subsurface flow inverse problems in sparse bases. Inverse Problems, 26:105016 (1-24), 2010.

[45] C. Serviere and P. Fabry. Blind source separation of noisy harmonic signals for rotating machine diagnosis. Journal of Sound and Vibration, 272:317-339, 2004. 
[46] P. Huang, J. Li, J. Sun, and J. Zhou. Vibration analysis in milling titanium alloy based on signal processing of cutting force. Int. J. Adv. Manuf. Technol., 64:613-621, 2013.

[47] Wolfgang Stephan. Total variation regularization for linear ill-posed inverse problems: Extensions and applications. $\mathrm{PhD}$ thesis, Arizona State University, 2008.

[48] J. J. Stickel. Data smoothing and numerical differentiation by a regularization method. Computers and Chemical Engineering, 34:467-475, 2010.

[49] C. L. Lawson. Contributions to the Theory of Linear Least Maximum Approximation. PhD Thesis, University of California, 1961.

[50] P. Rodriguez and B. Wohlberg. An iteratively weighted norm algorithm for total variation regularization. In Proceedings of the 2006 Asilomar Conference on Signals, Systems, and Computers, Pacific Grove, USA, 2006.

[51] A. Majumdar and R. K. Ward. Compressed sensing of color images. Signal Processing, 90:3122-3127, 2010.

[52] I. Daubechies, R. DeVore, M. Fornasier, and S. Gunturk. Iteratively re-weighted least squares minimization for sparse recovery. Commun. Pure Appl. Math., 63 (1):1-38, 2010.

[53] P. Rodriguez and B. Wohlberg. Efficient minimization method for a generalized total variation functional. IEEE Transactions on Image Processing, 18 (2):322-332, 2009. 
[54] R. A. Horn and C. R. Johnson. Topics in Matrix Analysis. Cambridge University Press, New York, 1991.

[55] P.C. Hansen. Regularization tools: A matlab package for analysis and solution of discrete ill-posed problems. Numerical Algorithms, 6:1-35, 1994.

[56] B. Qiao, X. Zhang, J. Gao, R. Liu, and X. Chen. Sparse deconvolution for large-scale ill-posed inverse problem of impact force reconstruction. Mechanical Systems and Signal Processing, 83:93-115, 2017. 